\title{
Social media and efficient computer infra- structure in smart city
}

\author{
Jerzy Balicki ${ }^{1,}$, Honorata Balicka $^{2}$, Piotr Dryja ${ }^{3}$, and Maciej Tyszka ${ }^{3}$ \\ ${ }^{1}$ Warsaw University of Technology, Faculty of Mathematics and Information Sciences, \\ 75 Koszykowa St., 00-662 Warsaw, Poland \\ ${ }^{2}$ Gdynia Maritime University, Faculty of Management and Commodity, 81-87 Morska St., 81-225 \\ Gdynia, Poland \\ ${ }^{3}$ Gdańsk University of Technology, Faculty of Electronics, Telecommunication and Informatics, \\ 11/12 Narutowicza St., 82-230 Gdańsk, Poland
}

\begin{abstract}
Social media require an efficient infrastructures of computer and communication systems to support a smart city. In a big city, there are several crucial dilemmas with a home and public space planning, a growing population, a global warming, carbon emissions, a lack of key resources like water and energy, and a traffic congestion. In a smart city, we expect an efficient and sustainable transportation, efficient management of resources and a better urban planning. In this paper, social media are proposed to support smart city with efficient computer infrastructure. Moreover, some methods are described to increase the availability and efficiency of an information infrastructure. Two criteria have been formulated to assign some key resources in a smart city system. The process of finding some compromise solutions from Pareto-optimal solutions has been illustrated. Metaheuristics of collective intelligence, including particle swarm optimization PSO, ant colony optimization ACO, an algorithm of bee colony $\mathrm{ABC}$, and differential evolution DE have been described due to smart city infrastructure improving. Other application of above metaheuristics in smart city have been also presented.
\end{abstract}

\section{Introduction}

Social media and an efficient city cloud are important for improving a live environment in a smart city by solving several crucial problems related to a traffic congestion, an urban planning, pollution emissions, warnings about bacteria in water or a lack of energy [1]. In the case of seaports, there is a dilemma of shortening the loading of containers. In turn, telemedicine can be very helpful in immediate treatment and even operation of seafarers and passengers on ships at sea [2]. To balance several infrastructure services in the smart city some criteria can be maximized. The first is the reliability of the city infrastructure of a computer distributed system. The second one is the probability that all critical tasks meet their deadlines.

\footnotetext{
* Corresponding author: J.Balicki@mini.pw.edu.pl
} 
Improving the quality of life and the required level of the economy can be achieved by means of more effective computerization of key processes related to the lives of residents of intelligent cities [3]. It is necessary to develop a new, more efficient infrastructure that we will use in a few years. At this time, however, you should efficiently revise existing infrastructure in order to improve its operation, including increasing the availability of 24 hours a day and 7 days a week. This involves taking into account the necessary upgrades, technical maintenance and software updates [4]. In addition, attacks on servers offering city services are, and probably will, unfortunately, become more intensive. What is more, the weakest element is always a user, an employee or an official who can provide sensitive data or passwords and, in a worse case, use their rights for their own purposes. A remedy for a number of the above dilemmas is a reliable IT system supporting the functioning of the city.

This paper is organized as follows. Section 2 discusses the urban tasks related to social media. Section 3 is dedicated for study a role of computer infrastructure in a smart city. In section 4, reliability of computer infrastructure in a smart city is characterized. Section 5 is devoted to the presentation of the time restrictions for city tasks. In section 6, some applications of metaheuristics for smart city are presented.

\section{City tasks in social media}

Social media and the Internet of Things can support a smart city to integrate a number of computers, sensors, actuators, software and data that are dedicated to manage information, give education, and improve safety [5]. Tasks can be related to labor, business, and commerce, too. Ten years ago, it was proved that departments of a city hall can use Facebook, Twitter, Instagram or Flickr for efficient communication between local government and citizens. Especially, some crisis situations like heavy snowstorm hits motivate citizens to frequent use the city's Facebook page to get information about a situation [6]. Moreover, photos can be viewed to analyze some cases. Social media sites are supposed to be connected to all city departments via an integrated social media center. Getting and sharing information from all the city's departments is very easy and some departments can receive feedback from citizens. Social media centers work in more effective way than a city phone hotline.

Social media can give an economic increase regarding an enlarged tourism traffic. Publicity generated by photos and advertising may attract tourists. Moreover, health care can be discussed via social media and some medical programs can be announced. Also, Twitter can be applied to gather data from some energy consumption sensors. Information about quality of city water can be presented, too. In crisis situation, some rescue operation can be supported during flood [7].

Social media is a new information channel between residents and departments of hall office about the current situation. Citizens can receive knowledge about the city, and they can communicate about each other. In results, decision making can be developed in many problems. A scale is large because in many countries over $70 \%$ population lives in cities [8].

Social media can be used to better understanding citizens by an identification some expectations of them. Moreover, social media can integrate several IT systems as well as real urban systems to share data with millions of citizens [9]. Balance between quality of many different systems is important. The network of connections between the domain systems and new ones is more important than the analysis of separately functioning urban systems. An advanced energy distribution system and a poor water system are not some good indicators of a smart city [10].

It may be happened that the construction of the airport will fail due to the lack of tourists, which is not prepared for a comfortable accommodation facilities, attractions to visit or ecological environment. Therefore, synergies should be achieved by balancing and 
cooperating between government, local government, education and business. Decisions should be the result of understanding the needs of residents, take into account their needs and preferences for decision-making criteria [11].

On the other hand, social media introduce some new tasks and some rules have to be prepared because some citizens can create many antagonistic remarks. Moreover, administrators of social media in a city department are supposed to cooperate with some experts to improve some social media sites [12]. Moreover, some additional meetings are required to study the effectiveness of social media, to apply new tools, to develop some brainstorming activity. However, social media has to be supported by Internet of Things as well as a cloud of computer resources [13].

\section{Computer infrastructure in a smart city}

From year to year, the number of projects related to smart cities is growing and extends. Also, the leading cities in Europe, such as Dublin or Amsterdam, have been implementing modern solutions in many seemingly unrelated spheres of urban life [14]. The smart city uses available information and communication solutions to improve the quality of life of residents. These can be infrastructure improvements, as an elastic traffic management system, and even electronic boards at bus stops. In such cases, the "intelligence" of the city is noticeable by the residents, because it directly affects the comfort of their lives [15].

In smart city, advanced ICT solutions (Information and Communication Technology) are used to protect the natural environment like monitoring the state of air pollution. Sometimes the definition of smart city takes into account aspects related to the operation of e-offices, which allows residents to efficiently perform administrative tasks over WWW and social media. The role of smart cities is to support sustainable development, improve the quality of life of their residents and improve administrative processes. An adequate example is Dublin, which is often considered a leader in smart city solutions. Few years ago, a mobile application for residents informing about important events in the area was introduced. These may include interruptions in water supply, road closures or events in the life of local community. Another solution is "intelligent dustbins" that send e-mails using a wireless connection to the Internet, as soon as the level of their use reaches $85 \%$ [16].

Smart city is based on the Internet of Things (IoT), which is a system that includes a set of devices that produce data and communicate with each other. IoT combines smartphones, cameras, sensors, motion sensors in clothes, as well as home devices like refrigerators informing you when stocks last. The range of IoT applications is almost unlimited, and one of them can be used to support communication in smart city systems. It is assumed that by 2020 , approximately 50 billion devices will be active under the IoT.

The amount of data necessary for processing is growing, and current technological solutions are no longer sufficient. It became necessary to redefine the methods that allow for the processing of huge data volumes in a satisfactory time. An important infrastructure from this point of view is an efficient infrastructure that enables fast, reliable data processing or provision of specific IT services for residents. It seems that this infrastructure should have minimal interruptions in work and be resistant to failures of its individual components. These, however, are inevitable for large-scale projects. In a city infrastructure, we can meet thousands of components.

Designing high availability city computer systems means one has to minimize the total time when the system is not accessible or unable to perform its tasks. The most popular technique to measure the availability is calculating the percent of time when system is fully operational (1).

$$
\text { availability }=\frac{\text { total_time }- \text { failure_time }}{\text { total_time }} \text {. }
$$


The value of $100 \%$ means that a system is failure proof. In practice, availability is sometimes described as "nines". Table 1 shows the availability values calculated for a smart city infrastructure for a 30 days period.

Table 1. Smart city infrastructure availability described as "nines".

\begin{tabular}{|c|c|c|}
\hline $\begin{array}{c}\text { Number of } \\
\text { „nines” }\end{array}$ & $\begin{array}{c}\text { Availability } \\
\text { percentage }\end{array}$ & Acceptable outage time \\
\hline 2 nines & $99 \%$ & 432 minutes $(1 \%$ from 43200 minutes $)$ \\
\hline 3 nines & $99,9 \%$ & 43 minutes $(0,1 \%$ from 43200 minutes $)$ \\
\hline 4 nines & $99,99 \%$ & 4 minutes $(0,01 \%$ from 43200 minutes $)$ \\
\hline
\end{tabular}

\section{Reliability of computer infrastructure in a smart city}

High available smart city system are designed by accommodating failure tolerance techniques to avoid the whole system downtime. One of most popular is to make a single component in a system redundant. In case of failure, a master component will be replaced by a secondary one and will take all responsibilities of its predecessor. For example, a power supply component or Internet provider may be redundant making the total failure more likely.

The self-repairing system is a crucial technique when dealing with high-availability systems. Self-repairing system can diagnose and fix itself automatically without human intervention. Given a "five nines" smart city system, it's often necessary to make it selfrepairing as a total acceptable time of failure is only 26 seconds in a month. It far too short time for a human to perform repair activities, but citizens require such wide access to some city services.

There are several methods which help to create a high available, self-repairing system. One of the most popular is a load-balancer service, which works as a proxy for requests coming to the system. A load-balancer redirects requests to only those components which are available. It helps to reduce the number of failed requests due to one of the components malfunctioning. Authors used this technique in the experimental Comcute PG grid at Gdańsk University of Technology. Comcute PG was designed to support crisis situations in the smart city agglomeration model.

Using virtualization machines VMW is another example of an applied method. It's responsible for monitoring nodes in a city cloud. Those nodes host virtual machines and when the failure occurs the supervised module is expected to perform necessary virtual machine's migrations. A virtual machine's downtime is significantly reduced when such a module is enabled for a city cloud.

Higher reliability usually means the system becomes more complex which in turn results in higher development and maintenance costs. Not all systems require the highest level of failure-tolerance. The hypothesis that $99,999 \%$ reliability is better than $99 \%$ reliability may be true only in a specified environment with a set of constraints applied. The decision about a level of reliability required for a system should be always made with respect to a particular system requirements.

Two computers performing some city tasks usually have a different reliability characteristics. Let the computation procedure $m_{v}$ be executed by the computer $\pi_{j} \in \Pi=$ $\left\{\pi_{1}, \ldots, \pi_{j}, \ldots, \pi_{j}\right\}$ during time $t_{v j}$. The computer $\pi_{j}$ be failed due to an exponential distribution with rate $\lambda_{j}$. Tasks should be executed on those computers where the smart city infrastructure reliability function $R$ is maximized: 


$$
R(x)=\prod_{v=1}^{V} \prod_{i=1}^{I} \prod_{j=1}^{J} \exp \left(-\lambda_{j} t_{v j} x_{v i}^{m} x_{i j}^{\pi}\right)
$$

where

$x_{i j}^{\pi}=\left\{\begin{array}{l}1 \text { if computer } \pi_{j} \text { is assigned to } w_{i}, \\ 0 \text { in the other case. }\end{array}\right.$

$x_{v i}^{m}=\left\{\begin{array}{l}1 \text { if task } T_{v} \text { is assigned to } w_{i}, \\ 0 \text { in the other case. }\end{array}\right.$

In order to maximize the reliability, it is essential to find some values of decision variables which is used to assign resources in the smart city nodes. Decision variables are presented as a pair of matrixes, as below:

$$
x=\left(X^{m}, X^{\pi}\right),
$$

where $X^{m}=\left[x_{v i}^{m}\right]_{V \times I}, X^{\pi}=\left[x_{i j}^{\pi}\right]_{I \times j}$.

It's a very challenging task to build a highly reliable smart city system. Figure 1 shows some reliability characteristics of two systems. The measure of reliability $\mathrm{R}$ decreases exponentially over time expressed in hours accordingly to formula (2). Let the first computer be failed once in a thousand hours on average. Then $\lambda_{1}=0.001$ [hour $\left.{ }^{-1}\right]$. On the other hand, the average time without failure for the second computer is five hundred hours, so the $\lambda_{2}=0.002\left[\right.$ hour $\left.^{-1}\right]$. When two computers are connected with the unfailing data transmission channel, the reliability of the system decreases much faster. A failure occurs three times in thousand hours in such a system.

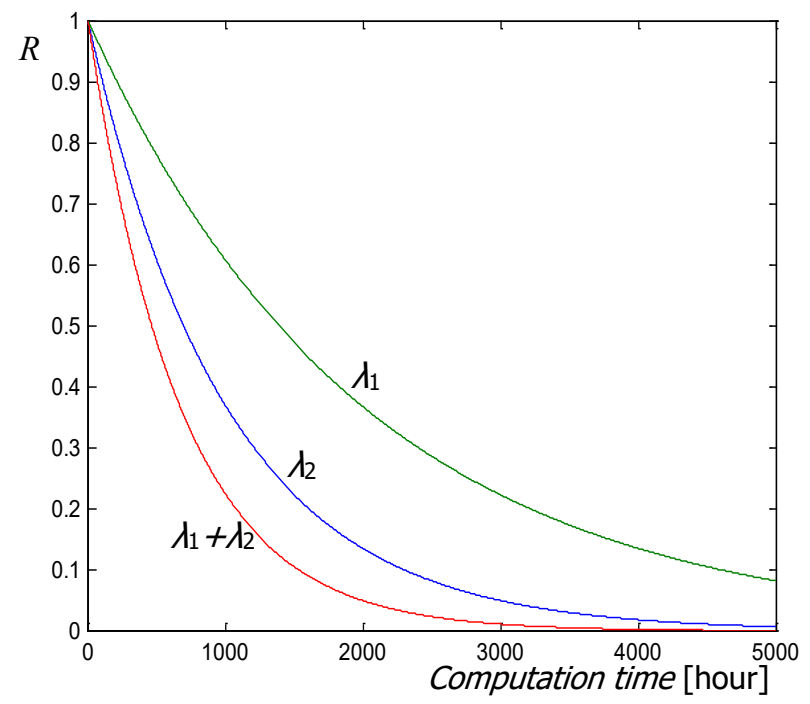

Fig. 1. Dependency between the availability and the computation time for two smart city systems.

There are thousands of components, including computers, sensors and actuators, in a system supporting a smart city computer infrastructure. For instance, the SmartSantander system, with over 20000 sensors is located in cities like Santander, Belgrade, Guildford and Lübeck. British project FixMyStreet, created to support citizen's complaints filing process has a similar complexity. In this system, citizens may file a complaint regarding noise, 
pollution, traffic jams etc. If this system is not accessible, frustration and disappointment become deeper and deeper.

A city with a highly available IT infrastructure was built in Japan. Smart city model in Tsukuba Science City permits on a deep analysis and a prediction of processes happening in such an intelligent agglomeration. The key findings from the experiment point to the role of citizens, quality of life, environmental research, education, economy, and health care, possibility of employment, mobility, infrastructure and technology. Those are the key areas which should be embraced and supported by well educated people, local communities and an unreliable ICT infrastructure.

\section{Time restrictions for city tasks}

In the smart city, there are several tasks that have to meet their deadlines. Delay of one task can cause delays of many tasks. Let $N$ city tasks in the 24-hour cycle be carried out in the smart city system. The task $T_{n}$ starts at $\alpha_{n}$ and should end at $\omega_{n}$. Figure 2 shows an example of dependency between two tasks. The first task performs calculations supporting the regulation of lights at intersections, and the second task - calculations for monitoring air pollution. In task $T_{1}$, after completing the procedure $m_{1}$, the $m_{2}$ operation is performed with the probability $q$ (serious accident at the intersection) in the OR decision subgroup (Fig. 2.) or operation $m_{3}$ - with the probability (1-q). However, in the task $T_{2}$, the procedure $m_{4}$ can be performed at most $L_{\max }$ times in the subgraph of the LOOP, where each iteration of the procedure is carried out with the probability $p$ (detection of pollution exceeding the safe level by the sensor).

It is easy to show that $2 L_{\max }$ of the instances are possible (Fig. 2.). The instance in which the $m_{2}$ procedure is executed once, and the procedure $m_{5}-k$ times, occurs with the following probability:

$$
p_{i}=q(1-p) p^{k-1}
$$

Based on the decisions of allocation of procedures to computers $\left(x^{m}, x^{\pi}\right)$, it is possible to estimate the completion times for calculations of procedures $C_{1}, \ldots, C_{v}, \ldots, C_{V}$ according to the adopted algorithm of task scheduling. Let $d_{v}$ represents the required deadline of the $v$ th procedure. If $C_{v} \leq d_{v}$, the deadline is not exceeded and the state of fulfillment of this restriction is set as $\xi\left(d_{v}-C_{v}\right)=1$. The states of constraints related to the deadlines of the procedures included in the $i$ th instance of the task execution diagram with the set of $M_{i}$ procedures can be written as follows:

$$
S_{i}=\prod_{m_{v} \in M_{i}} \xi\left(d_{v}-C_{v}(x)\right)
$$

As a consequence, we determine the probability of deadlines not being exceeded by procedures for all $K$ instances of the task implementation diagram in the smart city system, as follows:

$$
P_{D}(x)=\sum_{i=1}^{K} p_{i} \prod_{m_{v} \in M_{i}} \xi\left(d_{v}-C_{v}(x)\right)
$$




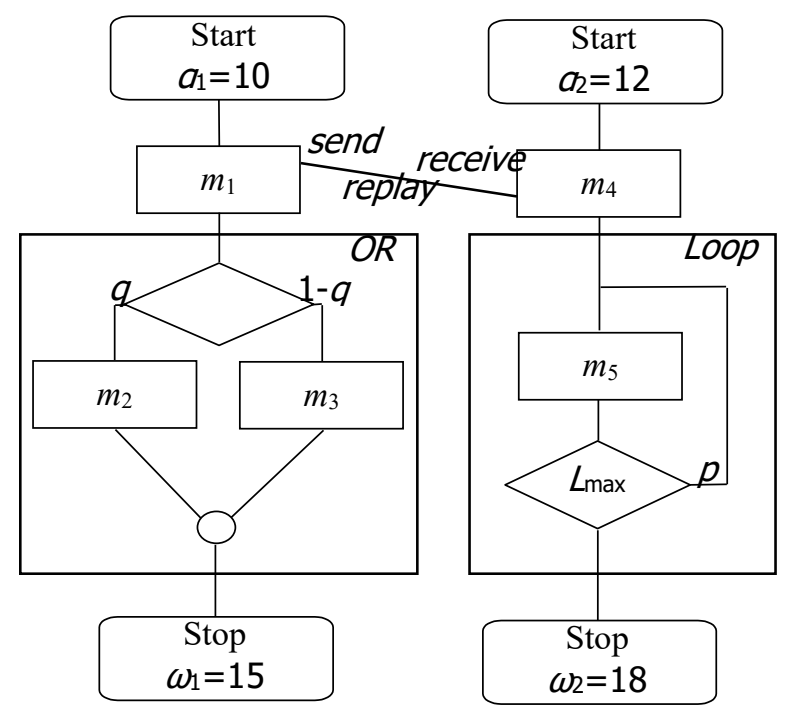

Fig. 2. An exemplary diagram of the implementation of two tasks in the smart city system

Fig. 3. shows a multi-criteria area of smart city infrastructure assessment. Marks were marked as pairs of values $\left(P_{D}, R\right)$ and restrictions were imposed on the optimization criteria. In addition, a compromise infrastructure was presented for the deployment of 14 procedures on 2 computers, which corresponds to the $y^{\mathrm{k}}$ rating nearest to the distance of Euclid to the ideal point $y^{\text {inf }}$. It was assumed that $P_{\mathrm{D}} \geq 0.6$ and $R \geq 0.3$.
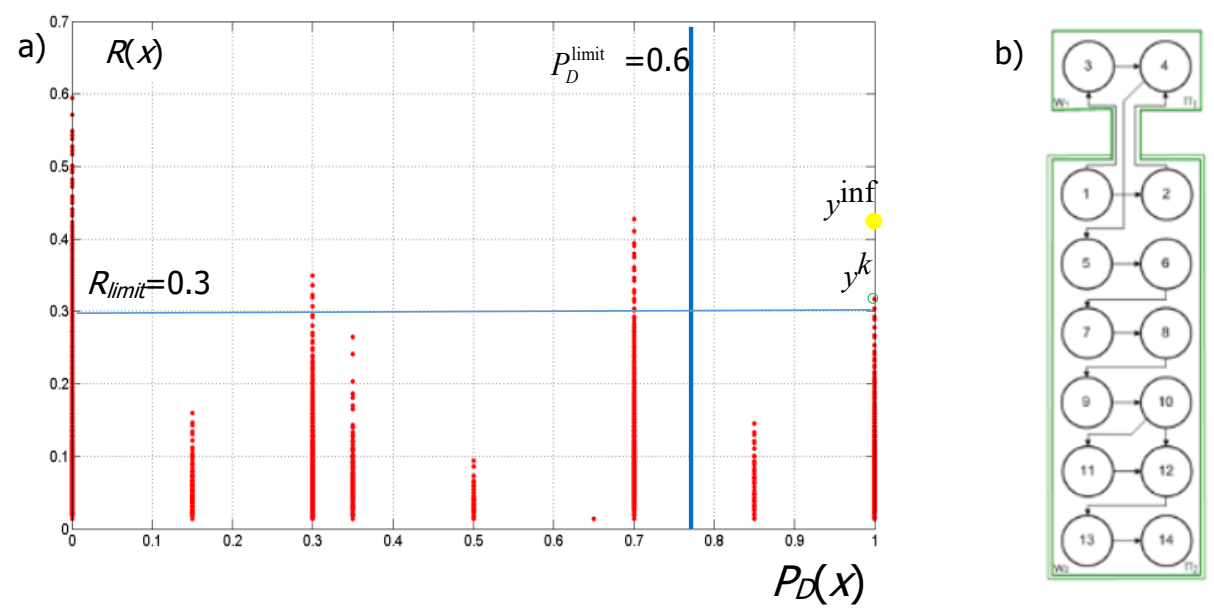

Fig. 3. Multicriteria space for assessing smart city IT infrastructures: a) ratings with restrictions on optimization criteria, b) compromise infrastructure

It is worth emphasizing that the values of imposed restrictions on criteria can be changed, and the stakeholders that have an impact on above decisions are: system administrators, designers, officials, local government, or even residents. On the basis of these restrictions, the set of admissible structures is narrowed, from which Pareto-optimal solutions can be chosen, and in particular the compromise infrastructure. It is worth emphasizing, however, that the problem is NP-hard due to computational complexity and metaheuristics are required to solve it. 


\section{Metaheuristics for smart city}

Metaheuristics are often very useful for IT infrastructure evaluation. Some of them such as Particle Swarm Optimization, Ant Colony Optimization and Artificial Bee Colony can be together referred to as swarm intelligence. Those algorithms share high-level characteristics. They usually consist of decentralized, self-organizing agent with a common operational knowledge. The ideas behind of all the three mentioned algorithms were heavily inspired by the nature: PSO - birds flock behavior, ACO - ant colony behavior, ABC - bee colony behavior.

The differential evolution DE algorithm is also worth mentioning. This algorithm was introduced as an optimization algorithm with a small number of parameters. The population consists of a certain number of vectors that contain variances to solve a problem. A characteristic feature of this algorithm is the crossing method which uses three vectors drawn from the population of parents. The selection of the best vector for the descendant population occurs between the vectors from the parent population and the vectors from the population created by using the crossing and mutation methods. In many optimization problems, the algorithm proved to be better compared to other evolutionary algorithms.

Bubal and Lee proposed planning of transport and communication connections in such a way that they are optimally adapted to the needs of people in large urban agglomerations, using the differential evolution algorithm [17]. The developed algorithm takes into account not only passengers' satisfaction, but also the costs incurred by bus, tram and train managers, so that the connections are profitable.

The Particle Swarm Optimization algorithm was constructed in 1995. Inspiration was the observation of social behaviors of flaps in the herd during their search for food. If a member of the herd senses the smell of food, it uses the sounds to inform others about its location. Then the rest of the herd tries to change the direction of the flight so as to get closer to it. If the next birds sense an even stronger smell of food, they again inform the remaining birds through even louder sounds. In this way they affect the direction of the flight of the rest of the herd.

Nikam and Mane have proposed using the PSO algorithm to optimize the wireless sensor network (WSN) connecting smart city infrastructure elements in the field of waste management [18]. WSN networks consist of many independent devices monitoring physical phenomena, such as temperature, movement or the presence of objects in space. Each node wirelessly transmits data to the central office where it is processed. The PSO algorithm has been used to improve network scalability, optimize energy consumption and resistance to damage.

ABC algorithm was introduced in 2005. Bees communicate and share information by so called waggle dance. It's a series of specific movements performed by a bee to share information about direction and distance to a food source. There are three distinct types of reactive agents when considering $\mathrm{ABC}$ algorithm: employed bees, onlookers bees and artificial food sources (solutions found for a particular optimization problem). Artificial bees, just like the real ones tend to share information in order to explore neighbor solutions. Those are often worth exploring as they may lead to even better ones. ABC algorithm proved to be a good choice in the field of combinatorial optimization as well as multi-objective optimization problems.

Fatnassi, Chebbi and Siala propose the ABC algorithm for solving Personal Rapid Transit (PRT) routing problem [19]. Authors used a modified version of algorithm to reduce the number of empty vehicle movements. A good computation time to solution quality ratio may be seen as key benefit when using it. PRT is an autonomous transportation system, often based on electric vehicles (pods) operating on previously prepared guideway network. A pod usually carries up to six passengers and run with the respect of calculated route. A computer 
decides which route is optimal in terms of travel time and energy consumption and minimize the risk of collisions. PRT was successfully introduced at the Heathrow airport in England as a partial replacement for bus transportation system.

Bello and Zeadally have proposed using the ACO algorithm to improve data transmission between IoT devices [20]. IoT networks can be characterized by a large variety of architectures, as well as significant dynamics of changes taking place, e.g. changing the topology of networks in the case of mobile devices. ACO can function as an important element to support routing in such a network. Thanks to the use of the algorithm, issues such as self-organization, self-configuration of the network, frequent change in the position of devices in the space or damage to damage can be solved [21].

\section{Remarks and conclusions}

The paper discusses the methods of increasing the availability and IT effectiveness of ininfrastructure in a smart city. Two criteria have been formulated to assess the distribution of key resources in the smart city system. The process of determining compromise solutions from Pareto-optimal solutions is illustrated. The metaheuristics of collective intelligence, including a swarm of particles, ants colonies, bee swarms and differential evolution with the help of which a smart city's compromise infrastructure can be defined, are discussed.

It is worth noting that the bee swarm algorithm was used to optimize the Personal Rapid Transit management system at Heathrow. In addition, the planning of transport and communication connections using the differential evolution algorithm takes into account not only the satisfaction of passengers, but also the costs borne by the managers of buses, trams and trains. In contrast, the particle swarm algorithm was used to optimize the wireless sensor network combining smart city infrastructure elements in the field of waste management. Ant colony algorithm is used to support routing in the Internet of Things, as well as selforganization and self-configuration IoT.

An interesting direction of further research is to construct a model and method including a broader perspective of resource optimization in a smart city.

\section{References}

1. R. Agarwal, A. Deo, S. Dasm, ACM SIGSOFT Softw. Eng. Notes 29, 2, $27-62$ (2004)

2. J. Balicki, Robot Motion and Control, 217-222 (RoMoCo, Poznań, Poland 2002)

3. R. Kanter, S. Litow, Informed and interconnected: A manifesto for smarter cities. Harvard Business School (2009)

4. J. Balicki, Computers, 417-422 (WSEAS, Rhodes, Greece, 2009)

5. A. Dukhanov, M. Karpova, K. Bochenina, Procedia Comput. Sc. 29, 2472-2482 (2014)

6. W. Gong, W. Wang, Cloud Computing and Intelligence Systems, 638-644 (IEEE, Beijing, 2011)

7. C. Hsu, H. Chen, K. Huang, Y. Huang, Comput. \& Math. Appl. 64, 5, 1506-1513 (2012)

8. S.V. Kolekar, S.G. Sanjeevi, D.S. Bormane, Computational Intelligence and Computing Research, 1-5 (IEEE, 2010)

9. J. Balicki, Z. Kitowski, Lect. Notes Comput. Sc. 1993, 373-384 (2001)

10. T. Kurilovas, I. Zilinskiene, V. Dagiene, Comput. Hum. Behav. 30, 550-557 (2014)

11. N. Pawar, S.K. Sonkar, Int. J. Em. T. Ad. Eng. 3, 7, 315-322 (2013)

12. M. Batty, K. Axhausen, F. Giannotti, A. Pozdnoukhov, A. Bazzani, M. Wachowicz, Eur. Phys. J-Spec Top 214, 481-518 (2012) 
13. J. Balicki, W. Korlub, J. Szymański, M. Zakidalski, Lect. Notes Comput. Sc. 8467, 771782 (2014)

14. M. Sayed, F. Baker, J. Softw. Eng. Appl. 8, 26-34 (2015)

15. J. Winiarski, Risk in business operations of enterprises (University of Gdańsk, 2014)

16. D. Xu, W. Huang, H. Wang, J. Heales, Inform. Manage. 51, 4, 430-440 (2014)

17. A.T. Bubal, L.S. Lee, J. Comput. Comm. 4, 12 - 14 (2016)

18. S. Nikam, P. Mane, Data Engineering and Communication Technology, 691-700 (2016)

19. E. Fatnassi, J. Chebbi, J. Siala, Evolutionary Computation (IEEE CEC, 2014)

20. O. Bello, S. Zeadally, IEEE Syst. J. 10, 3, 1172-1182 (2016)

21. J. Balicki, W. Korlub, H. Krawczyk, J. Paluszak, Human System Interactions, 271-278 (IEEE, Gdańsk, Poland, 2013) 